\title{
Rayleigh-Taylor instability in drop impact experiments
}

\author{
Victor Lherm $\odot,{ }^{1, *}$ Renaud Deguen, ${ }^{2}$ Thierry Alboussière $\odot,{ }^{1}$ and Maylis Landeau ${ }^{3}$ \\ ${ }^{1}$ LGL-TPE, CNRS, UJM, UCBL, ENSL, Université de Lyon, 69007 Lyon, France \\ ${ }^{2}$ ISTerre, Université Grenoble Alpes, Université Savoie Mont Blanc, CNRS, IRD, \\ Université Gustave Eiffel, 38000 Grenoble, France \\ ${ }^{3}$ Institut de Physique du Globe de Paris, CNRS, Université de Paris, 75005 Paris, France
}

(Received 4 August 2021; published 15 November 2021)

\begin{abstract}
This paper is associated with a video winner of a 2020 American Physical Society's Division of Fluid Dynamics (DFD) Gallery of Fluid Motion Award for work presented at the DFD Gallery of Fluid Motion. The original video is available online at the Gallery of Fluid Motion, https://doi.org/10.1103/APS.DFD.2020.GFM.V0019.
\end{abstract}

DOI: 10.1103/PhysRevFluids.6.110501

Consider a drop of radius $R_{d}$ and density $\rho_{d}$ falling on a pool of a liquid of density $\rho_{p}$. The drop strikes the pool surface at a velocity $v_{d}$, which results in the formation of a transient crater of mean radius $R(t)$. Though easy to conduct, this experiment hosts a wealth of fluid dynamics phenomena, involving a variety of hydrodynamical instabilities. It is also of interest in geology and planetology, drop impacts being in some respects surprisingly good analogs of crater-forming planetary impacts [1]. In most previous studies, the drop is made of the same liquid as the pool (see, e.g., [2-4]). New phenomena appear when the drop and pool liquids have different densities.

Figure 1 shows two snapshots obtained from experiments involving a drop whose density is either equal to $\left[\rho_{d} / \rho_{p}=1\right.$, Fig. 1(a)] or higher than $\left[\rho_{d} / \rho_{p}=1.8\right.$, Fig. 1(b)] that of the pool. In the experiment with $\rho_{d} / \rho_{p}=1.8$, the drop is made of a dense salt aqueous solution. We have also conducted a few experiments at $\rho_{d} / \rho_{p}=0.8$ using ethanol in the drop. The experiments are characterized by similar values of a modified Froude number $\mathrm{Fr}^{*}=\rho_{d} v_{d}^{2} / \rho_{p} g R_{d}$ and Bond number Bo $=\rho_{d} g R_{d}^{2} / \sigma$, where $g$ is the acceleration of gravity and $\sigma$ the surface tension. Here $\mathrm{Fr}^{*}$ is the parameter governing the maximum size of the crater. It can be interpreted as the ratio of the kinetic energy of the drop at impact (approximately $\rho_{d} R_{d}^{3} v_{d}$ ) to its gravitational potential energy just before impact (approximately $\rho_{d} g R_{d}^{4}$ ). In addition, Bo is the ratio of buoyancy forces to interfacial tension at the air-liquid interface. The two snapshots show the crater at the time at which its size is maximum.

Though the density ratio $\rho_{d} / \rho_{p}$ affects the shape of the crater and crown (Fig. 1), its most striking effect concerns the fate of the drop liquid. When $\rho_{d} / \rho_{p}=1$, the liquid from the drop is observed to spread at the floor of the crater, forming a layer whose thickness decreases with time in response to crater expansion, the liquid from the drop being redistributed over an increasingly large surface area. A similar behavior is obtained when $\rho_{d} / \rho_{p}<1$. The behavior is markedly different when $\rho_{d} / \rho_{p}>1$. In this case the layer formed from the drop liquid develops mushroom-shaped structures penetrating radially into the water pool [Fig. 1(b)]. Figures 2(a) and 2(b) show snapshots from

\footnotetext{
*victor.lherm@ens-lyon.fr

Published by the American Physical Society under the terms of the Creative Commons Attribution 4.0 International license. Further distribution of this work must maintain attribution to the author(s) and the published article's title, journal citation, and DOI.
} 


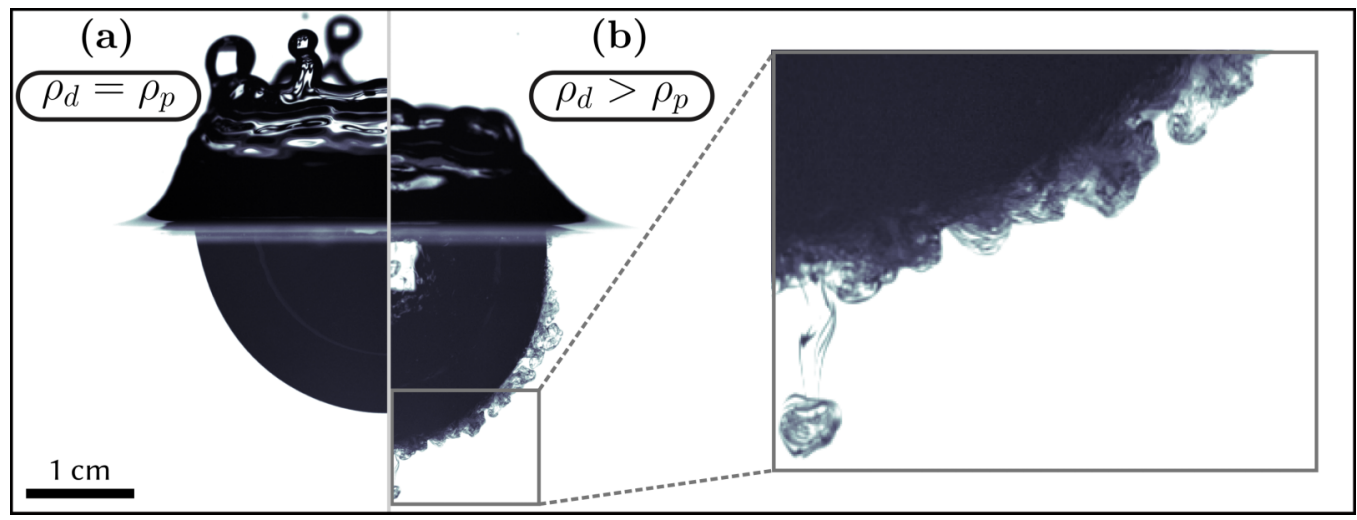

FIG. 1. Craters produced by the vertical impact of a drop onto a pool of water with (a) $\rho_{d} / \rho_{p}=1, \mathrm{Fr}^{*}=$ 977, and $\mathrm{Bo}=0.96$ and (b) $\rho_{d} / \rho_{p}=1.8, \mathrm{Fr}^{*}=976$, and $\mathrm{Bo}=1.34$. The original video is available online at https://doi.org/10.1103/APS.DFD.2020.GFM.V0019.

experiments using, respectively, direct visualization, with the drop liquid being dyed, and laserinduced fluorescence. Close inspection of these time sequences shows that the drop liquid first spreads over the crater floor to form a layer of approximately uniform thickness, before smallscale corrugations of the interface develop and gradually evolve into radially growing plumes. The fine-scale structure of the layer made of the drop liquid is reminiscent of mixing layers observed in Rayleigh-Taylor experiments in planar configurations (see, e.g., [5]). Measurements of the thickness of the mixing layer show that it first decreases with time before increasing until the crater reaches its maximum size.

These observations can be rationalized as follows. During crater opening, the rate at which the crater grows gradually decreases with time, which results in the deceleration of the boundary between the drop layer and the surrounding liquid. This situation is unstable with respect to Rayleigh-Taylor instability when the liquid in the layer is heavier than its surroundings. The deceleration $\dot{R}=d R / d t$ of the crater boundary can be estimated from conservation of energy (see, e.g., $[2,3])$. For a significant fraction of the crater opening phase, the energy balance is dominated by the kinetic energy of the flow surrounding the crater, which is approximately $\rho_{p} R^{3} \dot{R}^{2}$. Equating this with the impact kinetic energy, which is approximately $\rho_{d} R_{d}^{3} v_{d}^{2}$, and taking the derivative with respect to time gives $\ddot{R} \sim-g \operatorname{Fr}^{*}\left(R_{d} / R\right)^{4}$. When $\mathrm{Fr}^{*} \gg 1$, the deceleration can therefore be much larger than the acceleration of gravity. This explains why the dense liquid plumes grow radially

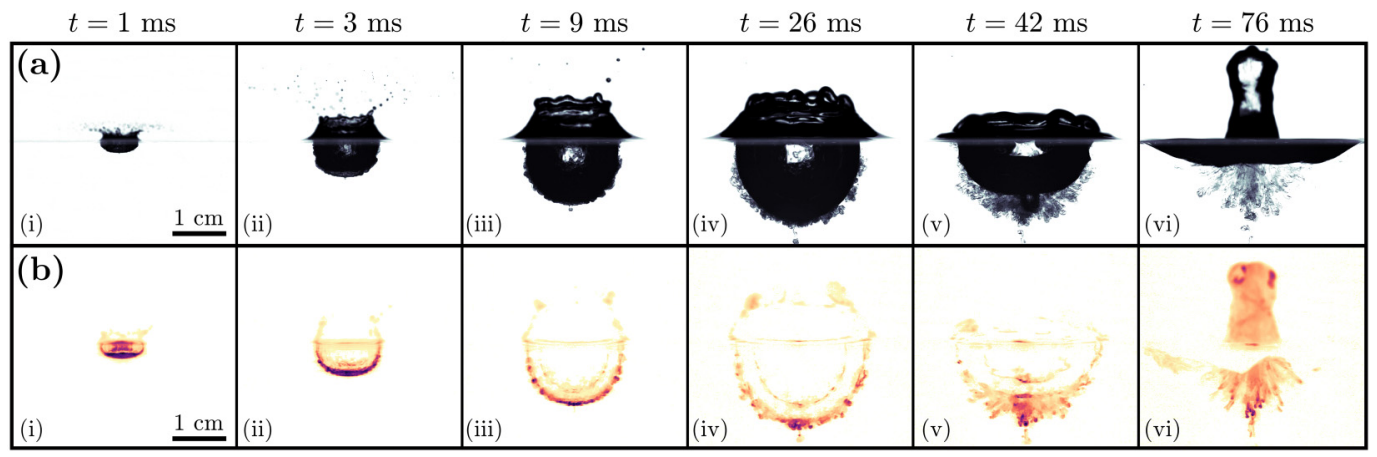

FIG. 2. Time sequences from experiments with $\rho_{d} / \rho_{p}=1.8$ using (a) direct imaging with a dyed liquid drop at $\mathrm{Fr}^{*}=976$ and $\mathrm{Bo}=1.34$ and (b) laser-induced fluorescence at $\mathrm{Fr}^{*}=1030$ and $\mathrm{Bo}=1.25$. 
outward rather than in the vertical direction. The nonmonotonic evolution of the mixing layer thickness results from a competition between the growth of the instability and the stretching of the layer associated with the expansion of the crater. The latter effect always dominates at early time, thus initially inducing a decrease of the layer thickness, but is overcome at later times if the density ratio is large enough.

This work was supported by the European Research Council under the European Union's Horizon 2020 research and innovation program (Grant No. 716429).

[1] M. Landeau, R. Deguen, D. Phillips, J. A. Neufeld, V. Lherm, and S. B. Dalziel, Metal-silicate mixing by large earth-forming impacts, Earth Planet. Sci. Lett. 564, 116888 (2021).

[2] O. G. Engel, Initial pressure, initial flow velocity, and the time dependence of crater depth in fluid impacts, J. Appl. Phys. 38, 3935 (1967).

[3] L. J. Leng, Splash formation by spherical drops, J. Fluid Mech. 427, 73 (2001).

[4] A. Bisighini, G. E. Cossali, C. Tropea, and I. V. Roisman, Crater evolution after the impact of a drop onto a semi-infinite liquid target, Phys. Rev. E 82, 036319 (2010).

[5] S. B. Dalziel, P. F. Linden, and D. L. Youngs, Self-similarity and internal structure of turbulence induced by Rayleigh-Taylor instability, J. Fluid Mech. 399, 1 (1999). 